\title{
A collaborative review process supports better outcomes from environmental modelling
}

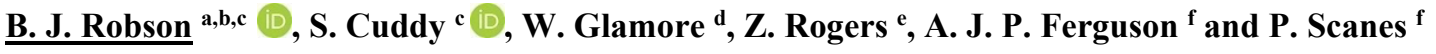 \\ ${ }^{a}$ Australian Institute of Marine Science, ${ }^{b}$ AIMS@JCU, ${ }^{c}$ CSIRO Land and Water, ${ }^{d}$ Water Research \\ Laboratory, School of Civil and Environmental Engineering, UNSW Sydney, ${ }^{e}$ Hunter Water, ${ }^{f}$ NSW \\ Department of Planning Industry and Environment \\ Email: b.robson@aims.gov.au
}

\begin{abstract}
Environmental modelling is used to support development planning, environmental policy, natural resource management, and environmental impact assessment. In all these contexts, it is important that the models have been carefully vetted and are suitable for the application at hand. Modelling clients often commission peer review by independent experts to ensure that the models that they rely upon are in line with best practise and can be defended when challenged.

Traditional review processes occur after model development and evaluation has been completed and documented. Here, we discuss the alternative of a collaborative peer review that is conducted alongside model design, development and evaluation, with regular communication between the client, review team and modelling team. The experiences of the UNSW modelling team, the CSIRO/AIMS review team and the client, Hunter Water, are each discussed separately.

Advantages of the collaborative review process included (for the review team) the opportunity to intervene early and suggest ways to avoid potential problems before too much had been committed to a particular approach, and reduced potential for conflict with the modelling team when concerns were raised; (for the modelling team) opportunity for collegiate open dialogue and the opportunity to understand critical issues for the reviewers and avoid wasting resources by guessing what reviewers would focus on; and (for the client) reduced potential for unpleasant surprises on review of the final models, and the opportunity to learn more about the modelling process to better inform their decisions relating to model development as well as their future use of the models.

Potential disadvantages included a more expensive review process (due to the requirement to engage the review team throughout the development process) and increased risk that the independence of the review process could be compromised if not carefully documented. In this case study, however, all parties were satisfied with the outcome.
\end{abstract}

Keywords: Review, receiving water modelling, water quality, best practice modelling 


\section{INTRODUCTION}

Environmental modelling is commonly used to provide information to inform urban and water resource planning and environmental policy (Harmel et al., 2014; Frassl et al., 2019). Decisions made in these contexts must balance the often-conflicting interests of multiple parties, so it is important that the models developed to support these decisions can withstand public scrutiny and potential legal challenge (Özkundakci, 2018). A key tool in quality assurance for environmental modelling for planning purposes is rigorous peer review, which can be achieved either through open publication of the models and their evaluation in internationally well-regarded scientific journals, or through reviews commissioned by the model client, or a combination of the two. For clients, commissioning a peer review ensures that the review will be completed in a timely fashion, whereas journal publication often occurs years after project completion (Verde Arregoitia and González-Suárez, 2019). Commissioned reviews also allow the client to choose who will conduct the review and gives the option of keeping details commercial-in-confidence.

Independent review of environmental models commissioned to support industry or government decision making should consider several factors. These include:

- Has the model development process followed established best practices, for instance by following the 'ten step' framework provided by Jakeman et al. (2006)?

- Is the model design and structure suitable for its intended application, taking into consideration the available data, the model's complexity, the available resources and the strength of biophysical understanding of the system being modelled (e.g. Robson, 2014a)?

- Is the model sufficiently supported by observational data?

- Has the model been properly evaluated using an appropriate suite of evaluation metrics and criteria (e.g. Bennett et al., 2013)?

- Is the model's performance appropriate to its intended application (Harmel et al., 2014)?

- Has the model and the associated data, metadata and software been adequately documented and appropriately stored (e.g. Schmolke et al., 2010)?

- Have the limitations of the model been transparently declared (Robson, 2014b)?

- Are the software, supporting data and model results available to the client in a format that will support their decision-making processes and future needs?

Where post hoc review reveals significant flaws in the modelling process or the final delivered models, there is considerable potential for conflict as it is likely that there has been substantial investment in the models and costs will be incurred by both the modelling team and client if significant revision is required or if the models are not suitable for their intended application.

An alternative to post hoc review is a collaborative review process in which independent reviewers are commissioned at the same time as the modelling team and review is conducted simultaneously with model development and evaluation. For large projects, the review team may be part of a steering committee that also includes representatives of the client and their major stakeholders. For smaller or mid-sized projects without a steering committee, a collaborative review process may still be beneficial.

In this paper, we describe a case study of a collaborative review process applied to development of hydrodynamic and water quality models to support decision-making regarding future wastewater treatment options and requirements for the Hunter Valley region.

\section{REVIEW PROCESS}

Hunter Water commissioned the University of New South Wales Water Research Laboratory (UNSW WRL) to develop hydrodynamic and water quality models for the Hunter River estuary, to inform planning of future wastewater treatment needs and priorities. NSW Office of Environment and Heritage (now NSW Department of Industry Planning and Environment) were contracted to conduct biogeochemical process studies in support of model development. With this contract in place, Hunter Water contracted CSIRO to provide independent review of the model (parts of which were subsequently subcontracted to the Australian Institute of Marine Science).

After reviewing the project inception documents to understand the purpose and intended scope of application of the models, the review team was brought in to assess the data to be used in model development and evaluation, the proposed modelling process, model design and model software, and to assess the final delivered models in terms of fitness-for-purpose. In between formal review steps, the review team, modelling team and 
Robson et al., A collaborative review process supports better outcomes from environmental modelling

client remained in communication to discuss options in addressing concerns and to provide advice to assist in trouble-shooting solutions.

Table 1 provides additional information regarding the steps undertaken by the client, the modelling team, and the review team at each stage of the model development process.

Table 1. Role of client, modelling team and review team at each stage of model development in the case study project. Although for the purposes of this paper, the process is divided into discrete steps, in practice, several of the steps overlapped and some discussions extended over a period of several months as the project progressed.

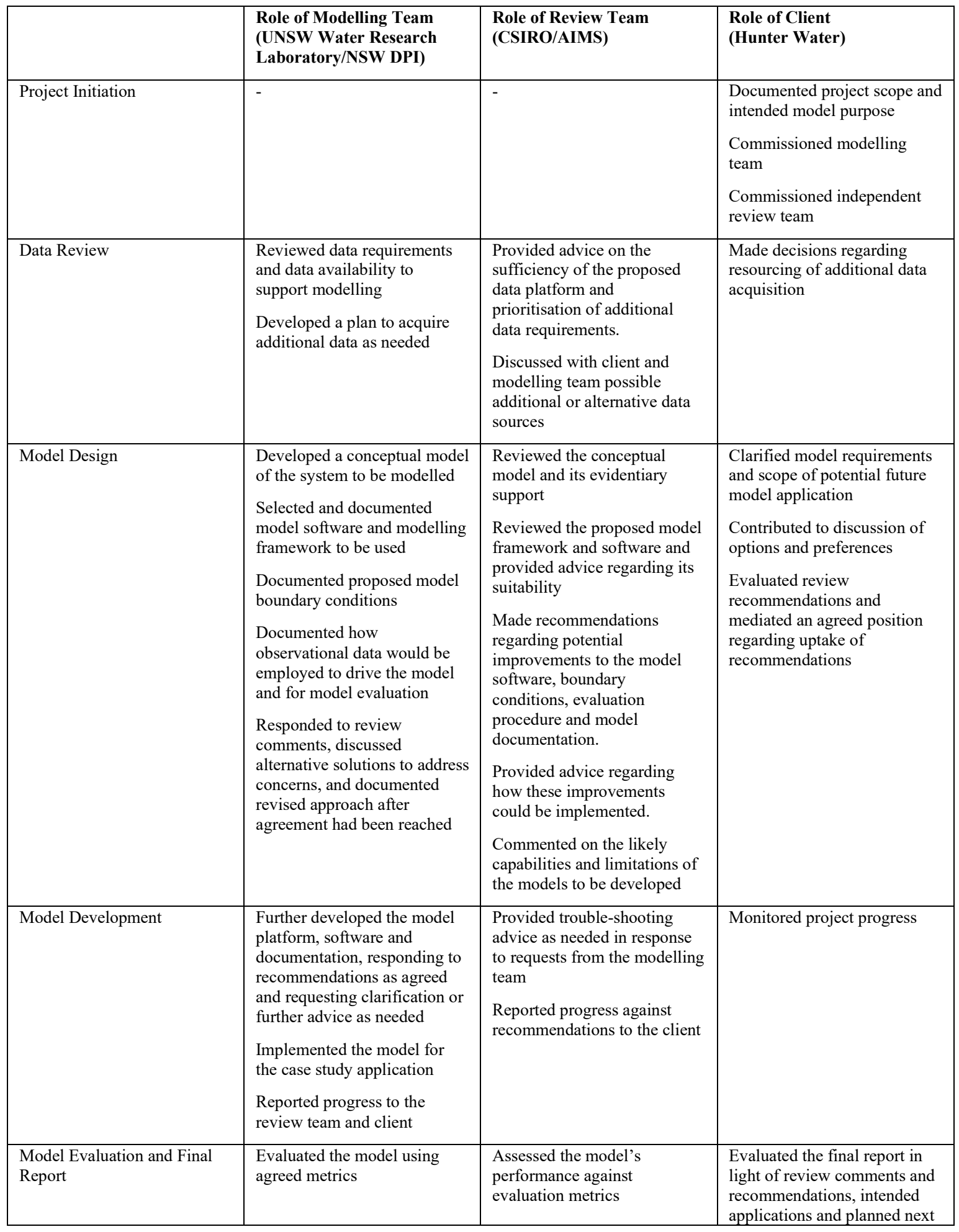




\begin{tabular}{|l|l|l|l|}
\hline & $\begin{array}{l}\text { Documented the model and } \\
\text { model evaluation results }\end{array}$ & $\begin{array}{l}\text { Made recommendations } \\
\text { regarding the capabilities and } \\
\text { limitations of the models and } \\
\text { their appropriate scope of } \\
\text { application }\end{array}$ & $\begin{array}{l}\text { steps, including scenario } \\
\text { development. }\end{array}$ \\
\hline
\end{tabular}

Hunter Water managed the process, organising workshops to bring together the modelling team, review team and client at each major stage. To ensure integrity of the process and maintenance of real and perceived independence, the modelling team submitted formal reports for review before each workshop, the modelling team submitted reports documenting their criticisms and assessment of the work after each workshop, and the modelling team formally reported its (agreed) response to criticisms.

\section{RESULTS AND DISCUSSION}

\subsection{Review team perspective [CSIRO and AIMS]}

Although this approach required a greater investment of time and consequently was more expensive than a post hoc model review, we believe it ultimately saved money and conflict and delivered a better outcome for the client. The collaborative review process gave us the opportunity to identify potential problems, especially in the model design and model evaluation protocols, before too much had been invested in the models. The modelling team was very open to feedback and worked with us to find solutions when concerns were raised. Where there was disagreement regarding how to proceed, the client, Hunter Water, was able to make a decision that prioritised their needs and considered the capabilities and limitations of the models to be developed.

One potential danger of this approach is that the independence of the reviewers could be compromised more easily than with an arms-length, post hoc review. We took care to ensure that this did not occur by documenting any concerns as they arose, while the modelling team documented their responses.

\subsection{Modelling team perspective [UNSW Water Research Laboratory]}

From the modelling team's perspective, it was extremely valuable to have early, continuous and collaborative input integrated into the project milestones. Despite the large project team with varied interest, the integrated review process provided a consistent yet alternative perspective that ensured the modelling team wasn't fixated on its own method or approach. Further, by having a collaborative review process throughout the project the modelling team was able to understand the critical issues for the reviewers and the entire group could modify our approach, remaining flexible, and avoid committing unnecessary resources to a task. The integrated process also removed the 'guess work' that surrounds typical peer review and ensured the typical 'masterslave' relationship didn't exist. Importantly, this promoted collegial and open dialogue with pre-eminent modellers who were willing to provide constructive feedback that could be immediately acted upon.

\subsection{Client perspective [Hunter Water]}

Hunter Water recognised the need for a parallel peer review process for the model development phases due to the complexity and scope of the model and the long timeframes involved. A post-hoc peer review would be less effective as there would be far less opportunity to revise model design and development. The success of the peer review process hinged on the extensive expertise of both the modelling and peer review teams and their capacity and willingness to work together. Of equal importance was the willingness of both parties to be flexible and adaptive in how and when the peer review was undertaken, as priorities and deadlines shifted. The peer review was a resounding success due the above factors, and also greatly assisted the Hunter Water project team in expanding their knowledge of aquatic modelling processes generally, and of the models used in particular.

\section{CONCLUSIONS}

A collaborative peer-review process provides considerable advantages over post hoc peer review in the case of commissioned environmental modelling projects. The approach is also applicable to other types of commissioned scientific studies. In the case study presented, all parties (the client, the modelling team and the review team) saw benefits from this approach. Like the concept of Open Review in review of journal papers (e.g. Ross-Hellauer et al., 2017), a collaborative review process supports discussion between reviewers and model developers to clarify recommendations, priorities and constraints and to facilitate an agreed solution to address concerns. In addition, because the collaborative review process begins before substantial investment has been made in model development and includes model clients (as next users or end users) as well as expert reviewers, it can reduce the potential for wasted effort and unsatisfactory outcomes. 
Because peer review is used not only to ensure quality but also to provide a defence against political or industry criticisms of models, careful management and full documentation of the review process is required to ensure that both the actual and perceived independence of the review team is maintained.

\section{ACKNOWLEDGEMENTS}

This work was funded by Hunter Water. Thanks to the anonymous reviewers for their helpful comments.

\section{REFERENCES}

Bennett, N. D., Croke, B.F.W., Guariso, G., Guillaume, J. H. A., Hamilton, S. H., Jakeman, A. J., MarsiliLibelli, S., Newham, L. T. H., Norton, J. P., Perrin, C. Pierce, S. A., Robson, B., Seppelt, R., Voinov, A. A., Fath, B. D. and Andreassian, A. (2013). Characterising performance of environmental models. Environmental Modelling \& Software, 40, 1-20.

Frassl, M. A., Abell, J. M., Botelho, D. A., Cinque, K., Gibbes, B. R., Jöhnk, K. D., Muraoka, K., Robson, B.J., Wolski, M., Xiao, M. and Hamilton, D. P. (2019). A short review of contemporary developments in aquatic ecosystem modelling of lakes and reservoirs. Environmental Modelling and Software, 117, 181-187.

Harmel, R. D., Smith, P. K., Migliaccio, K. W., Chaubey, I., Douglas-Mankin, K. R., Benham, B., Shukla, S., Muñoz-Carpena, R. and Robson, B. J. (2014). Evaluating, interpreting, and communicating performance of hydrologic/water quality models considering intended use: A review and recommendations. Environmental modelling \& software, 57, 40-51.

Jakeman, A. J., Letcher, R. A., and Norton, J. P. (2006). Ten iterative steps in development and evaluation of environmental models. Environmental Modelling \& Software, 21(5), 602-614.

Özkundakci, D., Wallace, P., Jones, H. F., Hunt, S., and Giles, H. (2018). Building a reliable evidence base: Legal challenges in environmental decision-making call for a more rigorous adoption of best practices in environmental modelling. Environmental science \& policy, 88, 52-62.

Robson, B. J. (2014). State of the art in modelling of phosphorus in aquatic systems: review, criticisms and commentary. Environmental Modelling \& Software, 61, 339-359.

Robson, B. J. (2014). When do aquatic systems models provide useful predictions, what is changing, and what is next? Environmental Modelling \& Software, 61, 287-296.

Ross-Hellauer, T., Deppe, A., and Schmidt, B. (2017). Survey on open peer review: Attitudes and experience amongst editors, authors and reviewers. PloS one, 12(12), e0189311.

Schmolke, A., Thorbek, P., DeAngelis, D. L., and Grimm, V. (2010). Ecological models supporting environmental decision making: a strategy for the future. Trends in ecology \& evolution, 25(8), 479-486.

Verde Arregoitia, L. D., and González-Suárez, M. (2019). From conference abstract to publication in the conservation science literature. Conservation Biology. 